\title{
Neural Apoptosis in the Retina during Experimental and Human Diabetes Early Onset and Effect of Insulin
}

\author{
Alistair J. Barber, ${ }^{\star}$ Erich Lieth, ${ }^{\ddagger}$ Sonny A. Khin, ${ }^{\star}$ David A. Antonetti,§ Adam G. Buchanan, ${ }^{\star}$ Thomas W. Gardner, ${ }^{\star \S}$ \\ and The Penn State Retina Research Group \\ *Department of Ophthalmology, ${ }^{\ddagger}$ Department of Neuroscience and Anatomy, and ${ }^{\S}$ Department of Cellular and Molecular Physiology, \\ Penn State University College of Medicine, Hershey, Pennsylvania 17033
}

\begin{abstract}
This study determined whether retinal degeneration during diabetes includes retinal neural cell apoptosis. Image analysis of retinal sections from streptozotocin (STZ) diabetic rats after 7.5 months of STZ diabetes identified $22 \%$ and $14 \%$ reductions in the thickness of the inner plexiform and inner nuclear layers, respectively $(P<0.001)$. The number of surviving ganglion cells was also reduced by $10 \%$ compared to controls $(P<0.001)$. In situ end labeling of DNA terminal dUTP nick end labeling (TUNEL) identified a 10fold increase in the frequency of retinal apoptosis in wholemounted rat retinas after $1,3,6$, and 12 months of diabetes ( $P<0.001, P<0.001, P<0.01$, and $P<0.01$, respectively). Most TUNEL-positive cells were not associated with blood vessels and did not colocalize with the endothelial cell-specific antigen, von Willebrand factor. Insulin implants significantly reduced the number of TUNEL-positive cells $(P<$ 0.05). The number of TUNEL-positive cells was also increased in retinas from humans with diabetes. These data indicate that retinal neural cell death occurs early in diabetes. This is the first quantitative report of an increase in neural cell apoptosis in the retina during diabetes, and indicates that neurodegeneration is an important component of diabetic retinopathy. (J. Clin Invest. 1998. 102:783-791.) Key words: cell death - diabetic retinopathy - retinal ganglion cells $\bullet$ neurons, neuroglia
\end{abstract}

\section{Introduction}

Diabetic retinopathy is the most common complication of diabetes, affecting over $90 \%$ of persons with diabetes and progressing to legal blindness in about $5 \%$. The vascular features of long-term diabetic retinopathy are well documented (1). Much less is known of pathological changes in retinal neurons that are likely to occur in diabetic retinopathy, given that the disease involves a progressive loss of visual function.

The pathologic vascular lesions associated with diabetic retinopathy have been described using the trypsin digest prepara-

Address correspondence to Thomas W. Gardner, The Ulerich Ophthalmology Research Center, Department of Ophthalmology, Penn State University College of Medicine, Hershey Medical Center, P.O. Box 850, Hershey, PA 17033. Phone: 717-531-6051; FAX: 717-5315475; E-mail: tgardner@psghs.edu

Received for publication 6 December 1997 and accepted in revised form 17 June 1998.

J. Clin. Invest.

(C) The American Society for Clinical Investigation, Inc. 0021-9738/98/08/0783/09 \$2.00

Volume 102, Number 4, August 1998, 783-791

http://www.jci.org tion (2), which removes the entire retinal parenchyma to expose the vasculature. Changes in the vasculature include microaneurysms, pericyte loss, basement membrane thickening, non-perfused acellular capillaries (1), and accelerated vascular cell death (3). The trypsin digest technique has advanced our understanding of the vascular lesions in diabetic retinopathy, but little attention has been paid to the neural cells (neurons and glia) of the retina. Nevertheless, results of several studies imply that the neural elements of the retina are also changed in diabetes. Glial cell proliferation is a well established late change in proliferative diabetic retinopathy and contributes to epiretinal membrane formation (4). Increased glial fibrillary acidic protein, a marker of central nervous system injury, and altered glutamate metabolism have also been noted recently in diabetic retinas $(5,6)$. Atrophy of ganglion cells and degeneration of the inner nuclear layer in the retinas of humans with diabetes was described by Wolter (7), who suggested that the gradual loss of neurons begins early in diabetes and that this degeneration may be a primary pathology that gives rise to vascular changes. Degeneration of the inner plexiform and ganglion cell layers was also described by Bloodworth in a histological study of 295 postmortem human eyes (8). The study described pyknosis and fragmentation of ganglion cell nuclei, features which are now recognized as typical characteristics of apoptosis. Interestingly, a lack of spatial correlation between ganglion cell loss and vascular lesions was observed. Furthermore, a study of the optic nerve in rats after 12 wk of streptozotocin diabetes found a reduction in the number of nerve fibers and an increased number of glial cells (9). Taken together, these data suggest that retinal neurodegeneration occurs in diabetic retinopathy.

Apoptosis has been identified as the mechanism of cell death in a number of degenerative diseases of the central nervous system (10-14). Apoptosis is an active process that has well described biochemical and morphological characteristics (15-17), including lack of an inflammatory response and DNA fragmentation caused by endonuclease activity (17). It is identified in histological sections by pyknotic nuclei, cytoplasmic condensation, and DNA fragmentation, which can be stained by in situ end labeling (TUNEL) ${ }^{1}$ of single or double strand breaks in DNA $(18,19,20)$. Apoptosis occurs in the retina in other degenerative diseases such as retinitis pigmentosa (21), anterior ischemic optic neuropathy (22), and glaucoma (23), but a mechanism of retinal neurodegeneration during diabetes has not been established.

In order to further investigate the pathology of diabetic retinopathy, two fundamental questions must be addressed: (1) when does retinal neurodegeneration begin with respect to the

1. Abbreviations used in this paper: STZ, streptozotocin; TUNELFITC, terminal dUTP nick end labeling-fluorescein-isothiocyanate; TUNEL-HRP, terminal dUTP nick end labeling-horseradish peroxidase. 
onset of diabetes; and (2) which cells, other than vascular cells, become apoptotic? The following study quantifies apoptosis in the retinas of streptozotocin diabetic rats and in postmortem retinas of humans with diabetes. We report that neural cell apoptosis is increased after 1 mo of diabetes and remains elevated over a 12-mo period. Furthermore, retinal apoptosis is reduced by insulin. There are overall reductions in the thickness of the inner layers of the retina, accompanied by a reduction in the number of ganglion cells in rat retinas after longterm experimental diabetes. These findings reveal that retinal neuron and glial cell degeneration occurs early on in the course of diabetes and should be considered an important component of the pathology of diabetic retinopathy.

\section{Methods}

Animals. Male Sprague-Dawley rats weighing 150-175 g were purchased from Charles River Laboratories (Wilmington, MA) and housed in the Penn State University College of Medicine animal facility in accordance with the Institutional Animal Care and Use Committee guidelines, and the Association of Research in Vision and Ophthalmology Resolution on the Care and Use of Laboratory Animals. All rats were group housed in suspended wire bottom cages with ad libitum food and water and under a normal 12-h light/dark schedule. Diabetes was induced by a single tail vein injection of streptozotocin (STZ; Sigma, St. Louis, MO) $(65 \mathrm{mg} / \mathrm{kg}$ freshly dissolved in citrate buffer, $\mathrm{pH} 4.5$ ) and confirmed three days later by urine glucose (Bayer reagent strips) and blood glucose $>250 \mathrm{mg} / \mathrm{dl}$ (Lifescan meter). Blood glucose was measured by the Lifescan meter method during late afternoon within $2 \mathrm{~d}$ of sacrifice, and by glycohemoglobin (Glycotest II kit; Pierce Chemical Co., Rockford, IL) at sacrifice. All rats were weighed weekly. Long-term diabetic rats were injected with $2 \mathrm{U}$ of insulin once a week after the second month of diabetes (subcutaneous Humulin Ultralente; Eli Lilly, Indianapolis, IN) to stabilize weight loss. Insulin-controlled diabetic rats were given small, solid phase subcutaneous implants (Linplant; Linshin, Canada) to deliver 2-4 $\mathrm{U}$ of bovine insulin/24 h. The implants were delivered below the skin through an $18 \mathrm{G}$ needle, under light anesthesia. No sutures were required and no bleeding or infection occurred. Rats were sacrificed under deep ether anesthesia followed by decapitation, and both eyes were enucleated immediately. The first eye was dissected to remove the whole retina, while the second was embedded in OCT and flash frozen in isopentane on dry ice. Eyes for the morphometric study were fixed by immersion in normal buffered formalin for $48-72 \mathrm{~h}$ and processed for paraffin embedding.

Histology and image analysis. Eyes from 7.5 mo diabetic $(n=6)$ and aged-matched control rats were analyzed. 16 sections $(6 \mu \mathrm{m})$ taken from the midpoint of each eye at approximately $40 \mu \mathrm{m}$ intervals were stained with thionin, a Nissl stain, to identify neuronal cell bodies (24). Images were captured using Optimas software on a PC computer linked to a high resolution video camera (DXC-960MD; Sony) mounted on a microscope (BH-2; Olympus, Melville, NY). Five visual fields were sampled from the posterior portion of each retina using a $40 \times$ objective (each visual field representing a $340 \mu \mathrm{m}$ length of retina). Within each visual field, the thickness of the inner plexiform layer, inner nuclear layer, and entire retina (excluding photoreceptors) was measured by the method of Hughes (25). To compensate for any bias due to skewed sectioning angle (i.e., deviation from $90^{\circ}$ ), all measurements were expressed relative to the thickness of the choroid in the respective section. The number of ganglion cells was also quantified in each visual field and the total count for the five sampled fields was expressed per $100 \mu \mathrm{m}$. Cells were categorized as ganglion cells only if they appeared in the ganglion cell layer and had large, round cell bodies containing positively-stained Nissl substance.

Terminal transferase dUTP nick end labeling (TUNEL). TUNEL was performed with horseradish peroxidase detection (TUNEL-HRP) in whole-mount retinas $(26,27)$, or with fluorescein detection (TUNELFITC) in $10 \mu \mathrm{m}$ cryostat sections. Isolated retinas were fixed in normal buffered formalin for $10 \mathrm{~min}$ at room temperature, and flatmounted on microscope slides coated with $2 \% 3$-aminopropyltriethoxy silane in humidified boxes, at $4^{\circ} \mathrm{C}$ overnight, before storing at $-20^{\circ} \mathrm{C}$. Before TUNEL-HRP staining, the retinas were rehydrated in PBS and fixed in $4 \%$ paraformaldehyde for $10 \mathrm{~min}$. The tissue was then dehydrated through graded alcohols, and defatted in xylene overnight to allow better penetration across the inner limiting membrane of the retina (27). The following day, the sections were rehydrated and permeabilized with $0.3 \%$ Triton for $15 \mathrm{~min}$, and digested with proteinase K (20 $\mu \mathrm{g} / \mathrm{ml}$, Sigma) for $30 \mathrm{~min}$. Endogenous peroxidase was quenched with $3 \%$ hydrogen peroxide for $10 \mathrm{~min}$. TUNELHRP staining was performed with the horseradish peroxidase Apoptag kit (Oncor Inc., Gaithersburg, MD), following the manufacturer's instructions. This kit uses terminal transferase to tail single or double strand $3^{\prime}-\mathrm{OH}$ ends of DNA with digoxigenin-11-dUTP and dATP, which is then recognized with an HRP-bound antidigoxigenin antibody. The HRP was reacted with $0.05 \% 3,3^{\prime}$-diaminobenzidine tetrahydrochloride (Gibco BRL, Gaithersburg, MD) and $0.03 \%$ hydrogen peroxide (Sigma, St. Louis, MO) to develop a brown precipitate that was easily observed in the whole-mount retinas. Each staining included a number of controls treated identically to the experimental tissue. The first positive control for TUNEL-HRP staining was a section of paraffin-embedded ventral prostate from a castrated rat (large-scale apoptosis occurs in the ventral prostate two days after castration) (28). This was compared to a similar section from an uncastrated adult rat. To provide a negative control, we also evaluated prostate sections from castrated rats by omitting terminal transferase. The second positive control, a whole-mounted ischemic retina, served as a comparison for neuronal apoptosis in whole-mounted retina. Retinal ischemia was induced in normal rats by raising the intraocular pressure of one eye to $100 \mathrm{mmHg}$ for $60 \mathrm{~min}$ with saline injected by a peristaltic micro-pump, causing the eye to blanch. This technique causes ganglion cell apoptosis for several days following reperfusion (28-30). TUNEL staining in the ischemic retina was compared to that in the contralateral (non-ischemic) retina. The three negative controls were: (1) sections of apoptotic prostate from which the terminal transferase enzyme was omitted; (2) sections from a normal prostate stained with TUNEL-HRP; and (3) the contralateral (non-ischemic) retina also stained with TUNEL-HRP.

For the colocalization study, TUNEL-FITC was used to stain 10$\mu \mathrm{m}$ cryostat sections simultaneously with a polyclonal antibody to von Willebrand factor (vWf, 1:500; Dako Corp., Carpinteria, CA). The TUNEL-FITC protocol was followed by an overnight incubation with anti-vWf in $10 \%$ goat serum with $0.1 \%$ Triton in PBS. The anti-vWf was detected with a rhodamine-conjugated donkey anti-rabbit IgG (1:600; Jackson ImmunoResearch Laboratories, West Grove, PA).

Postmortem eye specimens. Human eyes from anonymous donors were obtained from the National Disease Research Interchange (Philadelphia, PA). The following selection criteria were applied: $<65$ yr old, with either insulin-requiring diabetes or no diabetes, no lifesupport measures, and no chemotherapy. The donors were selected in pairs to be approximately age matched. The eyes were enucleated an average of $5.3 \pm 2.5 \mathrm{~h}$ after death, and preserved by immersion in $10 \%$ normal buffered formalin an average of $11.0 \pm 4.7 \mathrm{~h}$ after death. The interval from death to fixation was much shorter than that found to cause false positive TUNEL staining in central nervous system tissue (31). The eyes remained in formalin at room temperature for 1-2 wk before the retinas were dissected into quadrants by making four tangential cuts along the nasal, temporal, dorsal and ventral axes. The retinas were then washed for $2 \mathrm{~min}$ in PBS and two changes of reverse osmosis purified water, to remove excess salt. Further cuts were made in the radial edge of each quadrant to enable flat mounting on microscope slides coated with 3-aminopropyltriethoxy silane. The specimens were allowed to contact with the microscope slide for $24 \mathrm{~h}$ at $4^{\circ} \mathrm{C}$ and were then stained immediately by the TUNEL-HRP protocol as described above. 
Data collection and analysis. The percent weight change of rats was calculated as the difference between the weights at STZ injection and sacrifice, divided by the initial weight and multiplied by 100 . The blood glucose and percent weight change data were analyzed by Mann-Whitney or Kruskal-Wallis nonparametric tests. Analysis of the morphometry data was by ANOVA or unpaired $t$-test, where appropriate.

The total number of TUNEL-HRP-positive cells was recorded for each retina in a masked fashion. Each retina was visually scanned with a high power $(40 \times)$ objective in a serpentine manner to observe the entire retina. The number of TUNEL-HRP-positive cells was expressed per unit area, measured by tracing the outline of each retina viewed with a dissection microscope attached to the image analysis system described above. TUNEL-HRP-positive cell count data was $\log _{10}$ transformed to compensate for unequal group variances before analysis of variance. Individual differences between groups were measured using the Newmann-Keuls test.

\section{Results}

The retina degenerates during diabetes. To determine if atrophy and cell loss occur in the retina during diabetes, the relative thickness of the layers of the retina were measured in diabetic and age-matched control rats. Image analysis of serial sections of paraffin-embedded eyes from rats after 7.5 mo of STZ diabetes was used to measure changes in the thickness of the entire retina, the inner plexiform and nuclear layers, and to quantify the ganglion cell density. To standardize for bias introduced by skewed sectioning angles, all measurements were expressed relative to the thickness of the choroid in each section, which did not vary significantly with diabetes, averaging 101.5 $\pm 3.7 \mu \mathrm{m}$ in diabetic rats and $105.7 \pm 8.1 \mu \mathrm{m}$ in controls $(t-$ test, $t=0.47, P>0.6)$. The diabetic rats had a significantly reduced weight (percent weight change was 59.5 \pm 9.29 in the diabetic group and 154.3 \pm 13.47 in controls, $n=6, P<0.001)$ and
A

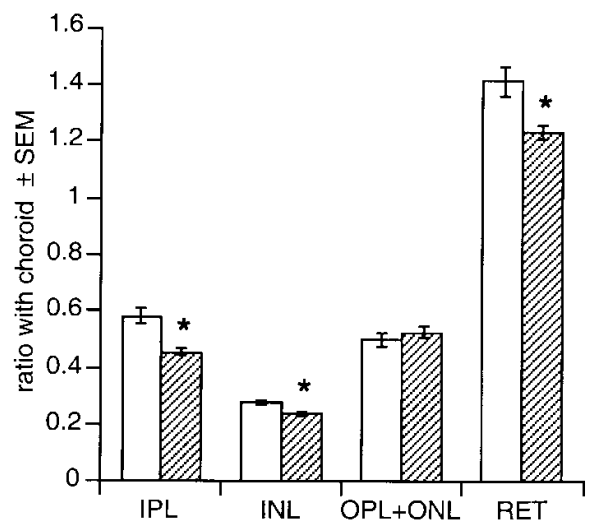

B

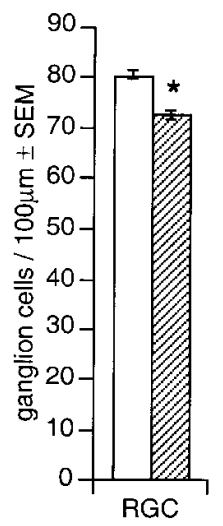

Figure 1. Atrophy of the rat retina after 7.5 months of diabetes. Various morphological parameters were measured by image analysis in 5 high power microscope fields along the retina in 16 sections from each of 6 diabetic and 6 control rat eyes. $(A)$ The average thickness of the inner plexiform layer $(I P L)$ and inner nuclear layer $(I N L)$ were reduced in diabetic rats (striped bars, $* P<0.001$ ), compared to controls (open bars). The combined thickness of the outer plexiform layer and outer nuclear layer $(O P L+O N L)$ was not different from control, but the overall thickness of the retina $(R E T)$ was significantly reduced in the diabetic rats $(P<0.001)$. $(B)$ There were significantly fewer retinal ganglion cells $(R G C)$ in diabetic rats (striped bars) compared to controls (open bars, $* P<0.001$ ). elevated blood glucose $(154.3 \pm 13.47 \mathrm{mg} / \mathrm{dl}$ in diabetic group and $65.3 \pm 4.73 \mathrm{mg} / \mathrm{dl}$ in controls, $P<0.001)$. The thickness of the inner plexiform layer was reduced by $22 \%$ of control and the inner nuclear layer was reduced by $14 \%$ of control, representing a significant reduction ( $t$-test, $P<0.001$ in both cases; Fig. $1 A$ ). The total thickness of the retina was also reduced by $13 \%$ of control. This reduction was completely accounted for by the reduced thickness in the inner plexiform and nuclear layers; the combined thickness of the outer plexiform and outer nuclear layers did not change $(t=0.775, P>0.4)$. The average ganglion cell density in the diabetic rats was $72.8 \pm 0.84$ / $100 \mu \mathrm{m}$, while that of controls was $80.5 \pm 1.19 / 100 \mu \mathrm{m}$ ( $t$-test, $P<0.001$; Fig. $1 \mathrm{~B}$ ), a $10 \%$ reduction. This reduction in the number of ganglion cell bodies, combined with the overall reduction in the thickness of the inner plexiform layer, indicates that there is a significant ganglion cell loss in the retina after 7.5 months of STZ diabetes. Additionally, the reduced thickness of the inner nuclear layer could be accounted for by the death of neurons and Müller cells. These data demonstrate that retinal degeneration occurs in diabetes and suggest that neural cells die at an accelerated rate compared to age-matched controls.

Retinal cell death occurs by apoptosis during diabetes. Morphometric analysis of sections of long-term diabetic rat eyes suggests that there is an accelerated rate of death of retinal ganglion cells, neurons, and possibly glial cells. To characterize and quantify this cell death, and to determine the time course of the increase, the number of apoptotic nuclei were counted in retinas after 1, 3, 6 and 12 mo of STZ diabetes. Because apoptotic cells are sparsely distributed in other retinal degenerations $(22,23)$, we stained whole-mount retinas using the TUNEL-HRP method to identify nuclei containing fragmented DNA, a characteristic of cells undergoing apoptosis, and the total number of TUNEL-HRP-positive cells in each retina was expressed per $0.5 \mathrm{~cm}^{2}$. This enabled an estimate of the number of apoptotic nuclei in the entire retina, and provided a superior sampling technique compared with TUNEL staining in histological sections.

Table I. Weight Change and Blood Glucose of Rats in the Apoptosis Study

\begin{tabular}{llcccc}
\hline $\begin{array}{l}\text { Duration of } \\
\text { diabetes (mo) }\end{array}$ & $\begin{array}{c}\text { Treatment } \\
\text { group }\end{array}$ & $n$ & $\begin{array}{c}\text { \% Weight } \\
\text { change } \pm \text { SEM }\end{array}$ & $\begin{array}{c}\text { Blood glucose } \\
\pm \text { SEM (mg/dL) }\end{array}$ & $\begin{array}{c}\% \text { Glycosylated } \\
\text { hb } \pm \text { SEM }\end{array}$ \\
\hline 1 & control & 6 & $26.7 \pm 1.58$ & $85.7 \pm 14.99$ & $6.2 \pm 2.43$ \\
& diabetic & 6 & $-11.3 \pm 4.61^{\ddagger}$ & $334.2 \pm 42.43^{\ddagger}$ & $14.1 \pm 1.05^{*}$ \\
3 & control & 6 & $72.2 \pm 4.18$ & $68.7 \pm 2.45$ & - \\
& diabetic & 6 & $-7.6 \pm 6.38^{\ddagger}$ & $328.7 \pm 6.9^{\ddagger}$ & - \\
6 & control & 6 & $114.7 \pm 6.80$ & $62.8 \pm 3.70$ & $6.0 \pm 0.99$ \\
12 & diabetic & 6 & $13.0 \pm 8.86^{\ddagger}$ & $242.5 \pm 37.8^{\ddagger}$ & $14.7 \pm 1.22^{\ddagger}$ \\
& control & 4 & $119.1 \pm 13.84$ & $52.8 \pm 3.64$ & - \\
& diabetic & 5 & $27.0 \pm 13.45^{*}$ & $201.6 \pm 29.06^{*}$ & -
\end{tabular}

Rats were injected with streptozotocin and housed alongside agematched controls for 1, 3, 6, and 12 months. \% Weight change and plasma glucose were measured as described under Methods. Glycosylated hemoglobin values were also measured in blood samples taken at sacrifice in the one and six month groups. The \% weight change, plasma glucose and glycosylated hemoglobin values were significantly different from control in all diabetic groups. ( ${ }^{*} P<0.05$, ${ }^{\ddagger} P<0.005$, Mann-Whitney test). 
A

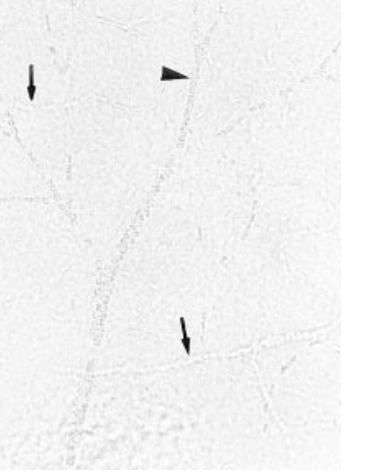

B

4

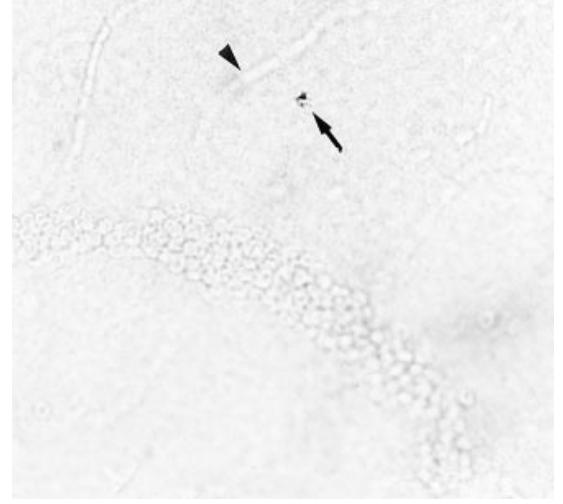

C

Figure 2. TUNEL-HRP-positive nuclei are pyknotic and not associated with vasculature. Whole-mounted retinas were stained for apoptotic cells using TUNEL-HRP. $(A)$ Normal whole-mounted rat retina. The structure and position of vasculature was easily visualized by phase contrast optics, both within the plane of focus (arrow head) and outside the plane of focus (arrows) $(\times 200)$. (B) Rat retina after 1 month of STZ diabetes, containing a TUNEL-HRP-positive cell with pyknotic nucleus (arrow) that is distinct from the surrounding vasculature. A small blood vessel (arrow head) is near the TUNEL-HRP-positive cell, but in a different focal plane $(\times 400)$. (C) Postmortem human retina after several years of diabetes, also containing a TUNEL-HRP-positive cell (arrow) $(\times 400)$.

All diabetic rats gained significantly less weight than their respective age-matched control groups (Table I). The blood glucose values of the diabetic rats were significantly higher than control values at all four time points $(P<0.05)$. Glycosylated hemoglobin, which is a measure of chronic hyperglycemia, was also significantly higher than control values in the diabetic groups at both time points measured.

TUNEL-HRP-positive cells were observed in retinas of diabetic rats (Fig. 2), indicated by a brown precipitate staining nuclei. These cells were easily recognized against negligible background staining and appeared identical to TUNEL-HRPpositive cells in both the ischemic retinas and the ventral prostate from castrated rats. In many instances, TUNEL-HRP pos- itive nuclei had a typical apoptotic morphology including pyknosis and nuclear fragmentation (Fig. 2). The vasculature of the retina could also be visualized with normal light field optics and was further resolved by differential interference contrast. The vast majority ( $>90 \%$ ) of TUNEL-HRP-positive cells were distinct from observable vasculature. TUNELHRP-positive cells appeared occasionally in all parts of the retina, mostly as individual cells or as loosely associated groups (Fig. 3) at different depths in the whole mounts, noted by focusing through the tissue.

Diabetic rats had significantly more TUNEL-HRP-positive cells at all four time points than their respective agematched controls (Fig. 4; 2-way ANOVA with post-hoc New-
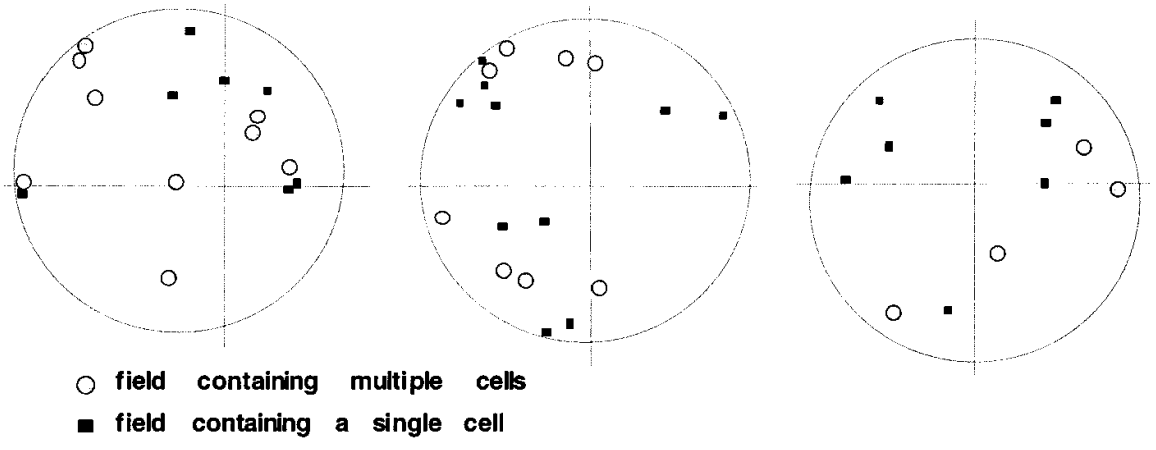

Figure 3. Distribution of TUNEL-HRP positive nuclei. The spatial distribution of TUNEL-HRP-positive nuclei was mapped in twelve retinas using microscope stage coordinates in the $\mathrm{x}$ and $\mathrm{y}$ plane, centered at the optic disc (intersect of $x-y$ axes). Plots of high power microscope fields containing single positive cells and groups of cells are shown for three representative retinas from rats after 1 and 3 mo of diabetes. Circles designate the approximate perimeter of each retina, which varied due to differences in dissection. Cells were seen in different focal planes, even within the same field, and did not appear as contiguous clusters. No consistent spatial distribution pattern was observed. 


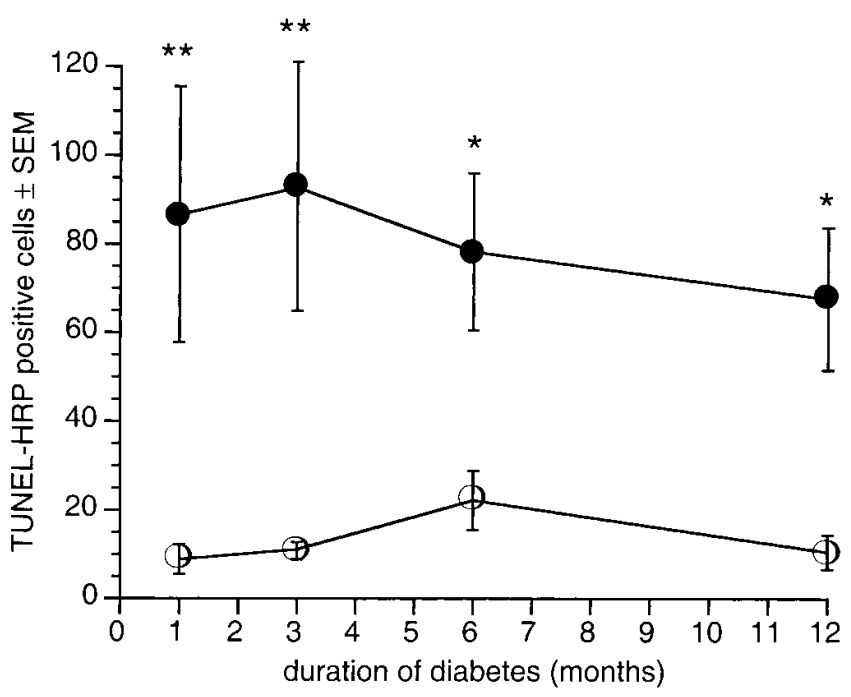

Figure 4. Apoptosis is increased in diabetic rat retinas. Rats were made diabetic by intravenous STZ injection and housed for between 1 and 12 months (black circles) alongside age-matched controls (white circles). Whole-mounted retinas were stained for apoptotic cells by the TUNEL-HRP method and the total number of positive cells was counted in each retina. The number of positive cells was expressed per $0.5 \mathrm{~cm}^{2}$. The diabetic rats had significantly more apoptotic cells compared to normal age-matched controls at all four time points $(* P<0.01, * * P<0.001$, ANOVA with Newmann-Keuls test).

mann-Keuls test, $P<0.001$ in 1 and 3 mo groups, $P<0.01$ in 6 and 12 mo groups), amounting to a $9-10$-fold increase. Therefore, the increase in cell death in diabetic rat retinas, as measured by morphometry (above), was attributed to apoptosis based on an increased number of TUNEL-HRP stained nuclei with pyknotic morphology. These data indicate that apoptosis is increased in the retinas of STZ diabetic rats as early as $1 \mathrm{mo}$ after the induction of experimental diabetes, and continues at the same frequency for at least 12 mo.

Apoptotic cells are distinct from the vasculature. Quantification by TUNEL staining found a large increase in apoptosis in diabetic rat retinas. The whole-mount TUNEL technique, however, is incompatible with immunohistochemical identification of cell types because of the extensive exposure to xylene. Morphometric measurements indicated that the number of surviving retinal ganglion cells is significantly reduced over a $7.5 \mathrm{mo}$ period of diabetes. Since apoptosis of vascular cells is established in diabetes (3), we sought to confirm that retinal apoptosis is not restricted to vascular endothelial cells.

TUNEL-FITC labeling was carried out in cryostat sections of retina simultaneously with immunohistochemical labeling of the vascular endothelial cell antigen, vWf. Tissue from both diabetic and control rats had similar degrees of positive vWf immunoreactivity and a small number of sections had positive TUNEL-FITC labeling (Fig. 5). TUNEL-FITC labeling was observed in the ganglion cell layer. These cells had large cell bodies characteristic of ganglion cells, and did not colocalize with vWf immunoreactivity. These data confirm that nonvascular cells, most likely ganglion cells, become apoptotic in the diabetic rat retina.

Insulin reduces apoptosis in the retina. To determine the effect of insulin on diabetes-induced retinal cell death during the first month of diabetes, rats were given subcutaneous insulin

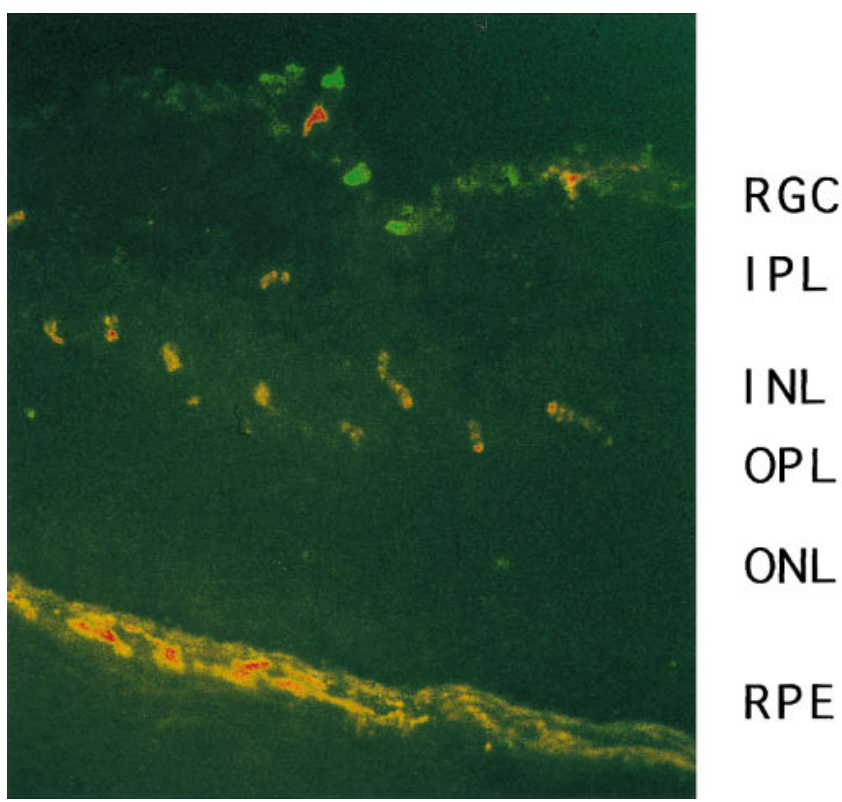

Figure 5. TUNEL positive cells are not vascular. Cryostat sections of rat retina were double labeled for the endothelial cell-specific antigen, von Willebrand factor (vWf, rhodamine label) and with TUNEL (fluorescein label). The ganglion cell layer of the retina contains a number of TUNEL-positive (green) cells that do not colocalize with vWf (orange-red) (Double exposure using fluorescein and rhodamine filter sets. Digital image reproduced from original $35 \mathrm{~mm}$ transparency, $\times 200)$. $R G C$, retinal ganglion cell layer; $I P L$, inner plexiform layer; $I N L$, inner nuclear layer; $O P L$, outer plexiform layer; $O N L$, outer nuclear layer; $R P E$, retinal pigment epithelial layer.

implants $3 \mathrm{~d}$ after STZ injection, and killed 1 mo later. The retinas were whole-mounted and stained with TUNEL-HRP as described earlier. The uncontrolled diabetic group (no insulin implants) gained significantly less weight $(P<0.001)$ and had higher blood glucose values than the control group $(P<0.0001)$ (Table II). The insulin-treated group had slightly elevated plasma glucose levels compared with controls $(P<0.05)$. Soon after the onset of diabetes, the average weight of the diabetic group became significantly less than that of the control group, while the insulin-treated group gained weight at the same rate

Table II. Weight Change and Glycosylated Hemoglobin of Rats in the Insulin Study

\begin{tabular}{|c|c|c|c|}
\hline Treatment group & $n$ & $\begin{array}{c}\% \text { Weight } \\
\text { change } \pm \text { SEM }\end{array}$ & $\begin{array}{c}\% \text { Glycosylated } \\
\text { hb } \pm \text { SEM }\end{array}$ \\
\hline Control & 12 & $27.51 \pm 0.92$ & $4.03 \pm 0.09$ \\
\hline Untreated diabetic & 12 & $-2.51 \pm 2.35 * *$ & $12.04 \pm 0.47^{\ddagger}$ \\
\hline Insulin-treated diabetic & 10 & $26.88 \pm 2.07$ & $6.93 \pm 0.46^{*}$ \\
\hline
\end{tabular}

The \% weight change of the insulin-treated diabetic group was not significantly different from control values, while that of the untreated diabetic group was less than the other groups $(* * P<0.0001$ with respect to both control and untreated diabetic groups, Kruskal-Wallis test with Dunn's multiple comparison). The \% glycosylated hemoglobin of the diabetic group was significantly higher than the control value ${ }^{\ddagger} P<$ $0.0001)$ while that of the insulin-treated group was significantly different from both the other groups $(* P<0.05)$. 
A

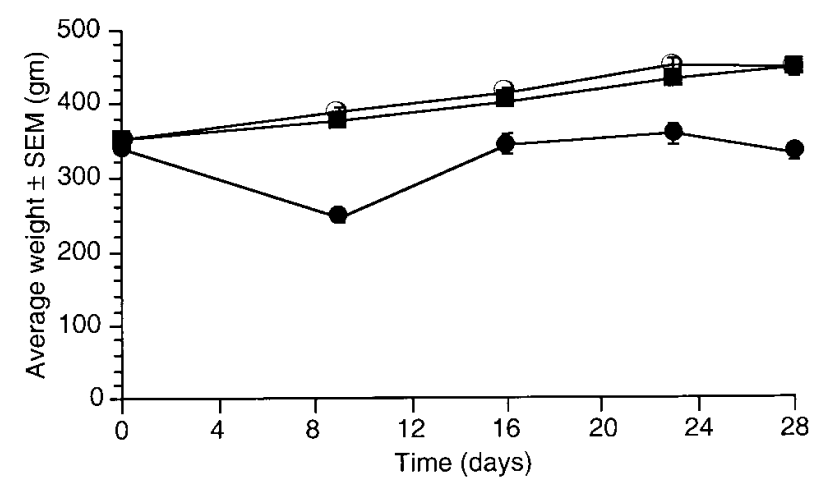

B

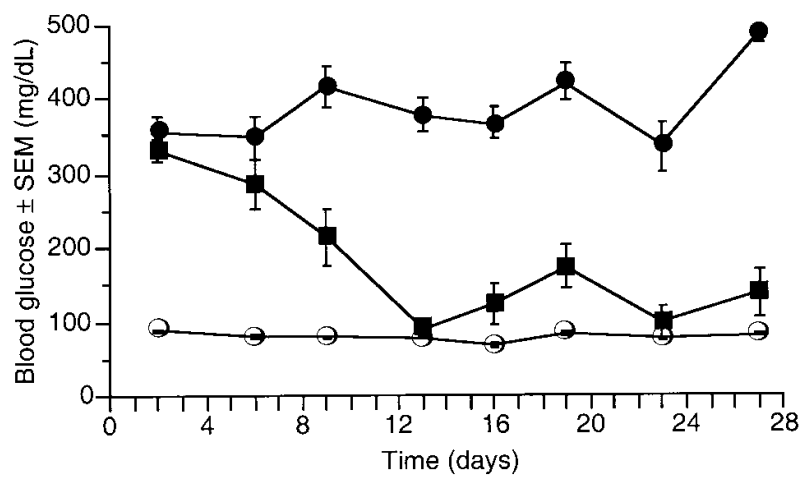

Figure 6. Insulin implants reduce blood glucose and stabilize weight loss in diabetic rats. Streptozotocin diabetic rats were given subcutaneous insulin implants for 1 month of diabetes. $(A)$ Diabetic rats lost weight (black circles) while age-matched controls (white circles) and diabetic rats with insulin implants (black squares) both gained weight at the same rate. $(B)$ Plasma glucose levels were elevated in the diabetic rats and normal in controls. The levels in diabetic rats with insulin implants gradually decreased to control levels over an initial $12 \mathrm{~d}$ period and remained close to normal for the duration of the experiment.

as control (Fig. $6 \mathrm{~A}$ ). The average blood glucose in the diabetic group was high from the onset of diabetes, but that of the insulin-treated group gradually reduced to control levels over a 12 -d period (Fig. $6 \mathrm{~B}$ ). As the insulin-treated rats gained weight, the number of implants given to each was increased to deliver the appropriate dose of insulin to maintain normoglycemia. The increase in the number of TUNEL-HRP-positive cells occurring in untreated diabetic rat retinas was consistent with our earlier findings (Fig. 7; $P<0.01$, compared to respective control group). The number of TUNEL-HRP-positive cells in the retinas of the insulin-treated rats was significantly less than in the diabetic group $(P<0.05)$, and not significantly greater than the control group. These data suggest that early insulin replacement reduces the number of apoptotic cells in the retina of the diabetic rat. The effect of insulin suggests that the increase in TUNEL-HRP reactivity is not due to a confounding effect of STZ injection, but is a direct consequence of diabetes.

Apoptosis is increased in the retinas of humans with diabetes. To determine if retinal apoptosis occurs to a similar degree in the retinas of humans with diabetes, we examined five

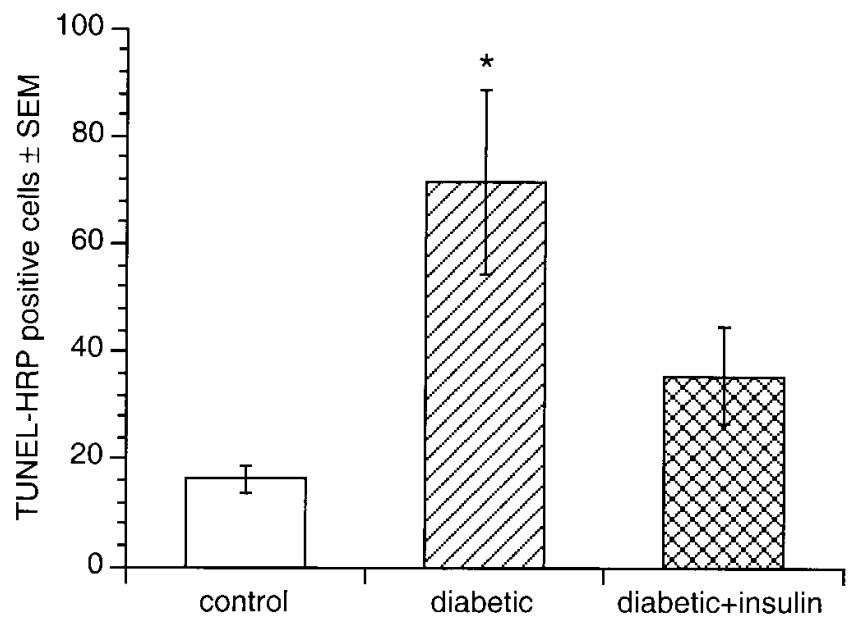

Figure 7. Insulin reduces retinal apoptosis in experimental diabetes. Whole-mount retinas were stained with TUNEL-HRP after 1 month of untreated diabetes (striped bar) or diabetes with insulin implants (crosshatched bar) and the number of TUNEL-HRP-positive nuclei compared to that of age-matched controls (white bar). The diabetic rats had significantly more TUNEL-HRP-positive cells than controls $\left({ }^{*} P<0.001\right)$ and the insulin treated group $(P<0.05)$.

postmortem human retinas from donors with and without diabetes. The retinas were divided into quadrants, flat-mounted, and labeled using the TUNEL-HRP protocol in the same way as the rat retinas in earlier experiments. Adhering retinal pigment epithelial cells were mostly washed off the specimens before flat-mounting, and the few remaining pigmented cells were easily identified by their position below the plane of the retina, and were not confused with TUNEL-HRP-positive cells. Table III summarizes the clinical details of each donor along with the number of apoptotic cells per $\mathrm{cm}^{2}$. Subjects 1, 2, and 3 had no history of diabetes, while Subjects 4 and 5 had confirmed histories of diabetes for 6 and $30 \mathrm{yr}$, respectively, and both used insulin. Subject 5 also had a confirmed history of diabetic retinopathy. The vasculature of each retina was examined with both light field and differential interference contrast optics for indications of abnormalities. The retina of Subject 5 contained many microaneurysms typical of diabetic retinopathy. The retina of Subject 3 also contained an area

Table III. Clinical Characteristics of Eye Donors and Number of TUNEL-HRP Positive Cells in Whole-Mounted Retinas

\begin{tabular}{|c|c|c|c|c|c|c|}
\hline Subjects & $\begin{array}{l}\text { Age } \\
(\mathrm{yr})\end{array}$ & Sex & $\begin{array}{c}\text { Diabetes } \\
\text { duration }(\mathrm{yr})\end{array}$ & $\begin{array}{c}\text { Retinal } \\
\text { pathology }\end{array}$ & $\begin{array}{c}\text { Cause of } \\
\text { death }\end{array}$ & $\begin{array}{c}\text { TUNEL-HRP } \\
\text { positive } \\
\text { cells } / 0.5 \mathrm{~cm}^{2}\end{array}$ \\
\hline 1 & 61 & M & - & none & CVA & 36.4 \\
\hline 2 & 54 & $\mathrm{~F}$ & - & none & CLD & 21.6 \\
\hline 3 & 43 & M & - & (BVO) & MI & 48.4 \\
\hline 4 & 62 & $\mathrm{~F}$ & 6 & no retinopathy & $\mathrm{CHF}$ & 62.2 \\
\hline 5 & 48 & M & 30 & NPDR & MI & 83.0 \\
\hline
\end{tabular}

Postmortem human retinas were examined for vascular abnormalities and stained for TUNEL-HRP positive cells as described. CVA, cerebrovascular accident; MI, myocardial infarction; CLD, chronic lung disease; CHF, congestive heart failure; NPDR, non-proliferative diabetic retinopathy; BVO, branch vein occlusion. 
characteristic of branch vein occlusion, in which vasculature was not clearly visible. No other vascular abnormalities were observed in the other specimens. Many TUNEL-HRP-positive cells had typical pyknotic morphology and were mostly distributed as single cells (Fig. 2). The subjects without diabetes had an average TUNEL-HRP-positive cell count of $70.9 \pm 21.9$ cells $/ \mathrm{cm}^{2}\left(58 \pm 14.8\right.$ cells $/ \mathrm{cm}^{2}$, excluding Subject 3), while the subjects with diabetes had an average of $145 \pm 20.8$ cells $/ \mathrm{cm}^{2}$. These data indicate that retinal apoptosis is a generalized response of retinal neural cells to diabetes and is not a specific feature of STZ-induced diabetes in rats. Furthermore, TUNEL-HRP-positive cells were observed away from microaneurysms in a human retina with diabetic retinopathy, and are therefore not limited to areas with retinal vascular lesions.

\section{Discussion}

This study reports that both experimental diabetes in rats and diabetes mellitus in humans are accompanied by increased apoptosis of retinal neural cells. The increase in the frequency of apoptosis occured after only 1 mo of experimental diabetes in rats, and a similar increase was noted in a human retina after six years of diabetes. The significant reduction of retinal ganglion cells and the reduction in the thickness of the inner plexiform and nuclear layers after 7.5 months of STZ diabetes suggest that the apoptotic cells are likely to include ganglion cells and other neurons. The histological location of TUNEL-positive cells and lack of colocalization with von Willebrand factor further suggest that neuronal and/or glial cell apoptosis occurs in the retina in diabetes. Viewed in the light of previous observations of neuronal cell death in diabetes (7-9), these data strongly suggest that neuronal cells begin to die soon after the onset of experimental diabetes in rats. They also confirm another brief observation of apoptosis in retinas of humans with diabetes, which were included in the control group of a study reporting apoptosis in open angle glaucoma (23). Therefore, neurodegeneration should be considered a component of diabetic retinopathy, as was suggested by both Wolter and Bloodworth $(7,8)$. Indeed, focal areas of retinal thinning have also been described in patients with diabetic retinopathy (32), and nerve fiber layer defects occur in patients with minimal or no vascular lesions (33).

Accelerated apoptosis of retinal vascular cells has been measured in trypsin-digested retinas from both rats and humans with diabetes (3), but the magnitude of apoptosis reported here indicates that most of the apoptotic cells in the retina are not endothelial cells or pericytes. Since quenching of endogenous peroxidase greatly reduced background staining associated with the vasculature and erythrocytes, TUNELHRP-positive cells were not confused with components of the blood. Furthermore, the increase in TUNEL-HRP staining is unlikely to be due to ischemic necrosis since it occurs after only one month of experimental diabetes in rats, before the onset of any known vascular occlusion. Neural cell death due to increased vascular permeability cannot be ruled out, however, since some increase in permeability can occur after one month of experimental diabetes in rats (34).

This study quantified the number of apoptotic cells in diabetic rat and human retinas by surveying whole-mount retinas. Other studies have observed some TUNEL-positive labeling in cryostat sections of diabetic rat eyes (35) and human retinas (23). Judging from the total number of apoptotic cells counted in the present study, it is unlikely that TUNEL staining of histological sections can be used to accurately quantify apoptosis in the diabetic retina, since a very large number of sections would have to be stained to make a reasonable sample. The section containing TUNEL-positive nuclei illustrated in Figure 5 was one of many radial sections that were found to contain no TUNEL-positive staining. This pattern of staining of radial sections is in line with the distribution of TUNEL-positive nuclei illustrated in Figure 3. It is clear that apoptosis also occurs in the normal retina, so in order to demonstrate an increase in apoptosis above baseline levels we sampled the entire retina.

Could the frequency of apoptosis explain the reduction in retinal thickness? The rat pup retina has $24.8 \pm 0.96$ million cells at postnatal day 8 , at which time the percentage of mitotic cells is negligible (36); so a reasonable estimate of the total number of retinal cells in the adult rats used here is $\sim 20$ million. Estimating the length of time that cells contain fragmented DNA as 6-12 h (37), the number of apoptotic cells measured here predicts a total loss of retinal cells of $1-10 \%$ over a 12 mo period. This is consistent with the morphometric changes we measured in the rat retinas after 7.5 months of diabetes. This degree of neural cell loss would not be detected clinically since the neural retina is normally transparent. The gradual nature of the cell death is consistent with the long latent period between the onset of diabetes and detectable diabetic retinopathy.

The increase in apoptotic cells in diabetic rats was mirrored in postmortem retinas from humans with diabetes. These data confirm that retinal neural cell death is not specific to STZ diabetes in rats. It is important to note that the number of apoptotic cells in both human retinas was about the same, but only one had detectable vascular lesions. The other donor was known to have had diabetes for only six years, and clinically evident vascular abnormalities do not usually develop during this short a duration of diabetes in humans. Therefore, these data suggest that retinal apoptosis is not a late consequence of vascular lesions.

Insulin delivered during the first month of diabetes reduced the number of apoptotic cells in the retina. These data suggest that retinal apoptosis in diabetes can be reversed by insulin therapy. Insulin may act by reversing some aspect of the physiology of diabetes (such as hyperglycemia). Indeed, galactosemic rats, which are hyperglycemic but not insulin deficient, also have increased vascular cell apoptosis (3). There is also extensive evidence that insulin can act as a trophic factor that affects cell survival in the central nervous system. Insulin reduces neuronal cell death in cerebral ischemia independently of blood glucose levels (38). Insulin regulates a number of factors associated with apoptosis, including neuronal intracellular calcium levels (39), calretinin and calbindin (40). Furthermore, insulin is a required survival factor in the central nervous system. At physiological concentrations, insulin rescues cultured optic nerve oligodendrocytes from death induced by trophic factor withdrawal (41), and is necessary for survival of retinal ganglion cells in culture (42). Insulin also rescues retinal vascular endothelial cells when apoptosis is induced by high glucose media (43). Regardless of the mechanism, these data strongly suggest that retinal apoptosis is reduced by exogenous insulin.

Secondary toxic factors may also contribute to retinal apoptosis in diabetes. Changes in glutamate metabolism occur in short-term experimental diabetes (6), implying that glial cells in the diabetic retina provide less protection from extracellular 
glutamate accumulation. An increase in the amount of unmetabolized glutamate in the retina may cause glutamate toxicity, which may in turn lead to neuronal apoptosis. Furthermore, retinal glial cells express more glial fibrillary acidic protein in early experimental diabetes (6) and in human diabetes (5), indicating a reactive state typical of central nervous system injury.

An increase in neural cell apoptosis could also contribute to microangiopathy because the apoptotic cells may include glia. Glial cells induce barrier properties in brain (44) and tight junction protein expression in retinal vascular endothelial cells (45). If retinal glial cells die, this could lead to a loss of endothelial cell barrier properties. Thus, retinal astrocyte or Müller cell death could impair barrier function. Indeed, other results from this laboratory show that the tight junction protein, occludin, is reduced in rat retinas after short-term STZ diabetes (Antonetti et al., manuscript submitted for publication).

This study addresses the two questions posed in the introduction. The data suggest that: (1) apoptosis begins soon after the onset of diabetes and continues at a relatively constant rate; and (2) the apoptotic cells include ganglion cells and other neural cells. Removal of insulin by STZ gives rise to neural cell death in the retina, but it is not yet established whether this relationship is a direct one or if the loss of insulin gives rise to apoptosis by an indirect mechanism, such as hyperglycemia. In light of these data, we suggest that diabetic retinopathy should be viewed as a disease that includes retinal neurodegeneration as well as vascular pathologies. Further attempts to understand and treat this disease must include a consideration of the roles of all three cellular elements (neurons, glial and vascular cells), and how they interact under the physiological constraints of diabetes.

\section{Acknowledgments}

The authors thank Mr. and Mrs. Jack Turner, Athens, GA, for their generous support. T.W. Gardner is an Established Investigator of the American Heart Association. We also thank Dr. Sarah Bronson for her suggestions during preparation of the manuscript. Lifescan reagent strips were generously provided by Lifescan Inc.

This work was supported by grants from the Pennsylvania Lions Sight Conservation and Eye Research Foundation, the Juvenile Diabetes Foundation International (\#197038 EL, \#196033 TWG) and the National Institutes of Health (K11 EY00331).

\section{References}

1. Engerman, R.L., and T.S. Kern. 1995. Retinopathy in animal models of diabetes. Diabetes Metab. Rev. 11:109-120.

2. Kuwabara, T., and D.G. Cogan. 1960. Studies of retinal vascular patterns. Part 1. Normal architecture. Arch. Ophthalmol. 64:904-911.

3. Mizutani, M., T. Kern, and M. Lorenzi. 1996. Accelerated death of retinal microvascular cells in human and experimental diabetic retinopathy. J. Clin. Invest. 97:2883-2890.

4. Ohira, A., and E. de Juan. 1990. Characterization of glial involvement in proliferative diabetic retinopathy. Ophthalmologica. 210:187-195.

5. Mizutani, M., C. Gerhardinger, and M. Lorenzi. 1998. Müller cell changes in human diabetic retinopathy. Diabetes. 47:445-449.

6. Lieth, E., A.J. Barber, B. Xu, C. Dice, M.J. Ratz, D. Tanase, J.M. Strother, and the Penn State Retina Research Group. 1998. Glial reactivity and impaired glutamate metabolism in short-term experimental diabetic retinopathy. Diabetes. 47:815-820.

7. Wolter, J.R. 1961. Diabetic retinopathy. Am. J. Ophthalmol. 51:11231139.

8. Bloodworth, J.M.B. 1962. Diabetic retinopathy. Diabetes. 2:1-22.

9. Scott, T.M., J. Foote, B. Peat, and G. Galway. 1986. Vascular and neural changes in the rat optic nerve following induction of diabetes with streptozoto- cin. J. Anat. 144:145-152.

10. Su, J.H., A.J. Anderson, B.J. Cummings, and C.W. Cotman. 1994. Immunohistochemical evidence for DNA fragmentation in neurons in the AD brain. NeuroReport. 5:2529-2533.

11. Mochizuki, H., K. Gotot, H. Mori, and Y. Mizuno. 1996. Histochemical detection of apoptosis in Parkinson's disease. J. Neurol. Sci. 137:120-123.

12. Petito, C.K., and B. Roberts. 1995. Evidence of apoptotic cell death in HIV encephalitis. Am. J. Pathol. 146:1121-1130.

13. Anderson, A.J., J.H. Su, and C.W. Cotman. 1996. DNA damage and apoptosis in Alzheimer's disease: colocalization with c-jun immunoreactivity, relationship to brain area, and effect of postmortem delay. J. Neurosci. 16:17101719.

14. Chalmers-Redman, R.M.E., A.D. Fraser, W.Y.H. Ju, J. Wadia, N.A Tatton, and W.G. Tatton. 1997. Mechanisms of nerve cell death: apoptosis or necrosis after cerebral ischemia. In Neuroprotective Agents and Cerebral Ischemia. International Rev. of Neurobiol. A.R. Green and A.J. Cross, editors. Academic Press, San Diego, CA. 40:1-25.

15. Kerr, J.F., A.H. Wyllie, and A.R. Currie. 1972. Apoptosis: a basic biological phenomenon with wide-ranging implications in tissue kinetics. $\mathrm{Br} . J$. Cancer. 26:239-257.

16. Wyllie, A.H., R.G. Morris, A.L. Smith, and D. Dunlop. 1980. Cell death: the significance of apoptosis. Int. Rev. Cytol. 68:251-306.

17. Majno, G., and I. Joris. 1995. Apoptosis, oncosis, and necrosis: an overview of cell death. Am. J. Pathol. 146:3-15.

18. Gavrielli, Y., Y. Sherman, and S.A. Ben-Sasson. 1992. Identification of programmed cell death in situ via specific labeling of nuclear DNA fragmentation. J. Cell Biol. 119:493-501.

19. Iseki, S. 1986. DNA strand breaks in rat tissues as detected by in situ nick translation. Exp. Cell Res. 167:311-326.

20. Wijsman, J.H., R.R. Jonker, R. Keijzer, C.J.H. Van De Velde, C.J. Cornelisse, and J.H. Van Dierendonck. 1993. A new method to detect apoptosis in paraffin sections: in situ end-labeling of fragmented DNA. J. Histochem. Cytochem. 41:7-12.

21. Wong, P. 1994. Apoptosis, retinitis pigmentosa, and degeneration. Biochem. Cell Biol. 72:489-498.

22. Levin, L.A., and A. Louhab. 1996. Apoptosis of retinal ganglion cells in anterior ischemic optic neuropathy. Arch. Ophthalmol. 114:488-491.

23. Kerrigan, L.A., D.J. Zack, H.A. Quigley, S.D. Smith, and M.E. Pease. 1997. TUNEL-positive ganglion cells in human primary open-angle glaucoma. Arch. Ophthalmol. 115:1031-1035.

24. Sheehan, D.C., and B.B. Hrapchak. 1987. Theory and practice of histotechnology. Batelle Press, Columbus, Ohio. 253 pp.

25. Hughes, F.W. 1991. Quantitation of ischemic damage in the rat retina. Exp. Eye. Res. 53:573-582.

26. Bien, A., M.F. Humphrey, C. Seidenbecher, B.A. Sabel, and M.R Kreutz. 1996. Apoptosis in retinal ganglion cells after a controlled crush of the adult optic nerve. Soc. Neurosci. 22:126.2. (Abstr.)

27. Versaux-Botteri, C., E. Martin-Martinelli, J. Nguyen-Legros, M. Geffard, A. Vigny, and L. Denoroy. 1986. Regional specialization of the rat retina: catecholamine-containing amacrine cell characterization and distribution. $J$. Comp. Neurol. 243:422-433.

28. Kyprianou, N., H.F. English, and J.T. Isaacs. 1988. Activation of a $\mathrm{Ca}^{2+}$ $\mathrm{Mg}^{2+}$-dependent endonuclease as an early event in castration-induced prostatic cell death. Prostate. 13:103-117.

29. Buchi, E.R. 1992. Cell death in the rat retina after a pressure-induced ischaemia-reperfusion insult: an electron microscopic study. I. Ganglion cell layer and inner nuclear layer. Exp. Eye Res. 55:605-613.

30. Kuroiwa, S., N. Katai, H. Shibuki, T. Kurokawa, J. Umidira, T. Nikaido, K. Kametani, and N. Yoshimura. 1998. Expression of cell cycle-related genes in dying cells in retinal ischemic injury. Inv. Ophthalmol. Vis. Sci. 39:610-617.

31. Petito, C.K., and B. Roberts. 1995. Effect of postmortem interval in in situ end-labeling of DNA oligonucleosomes. J. Neuropath. Exp. Neurol. 54 761-765.

32. Gardner, T.W., M.L. Miller, D. Cunningham, and G.W. Blankenship. 1995. The retinal depression sign in diabetic retinopathy. Graefe's Arch. Clin. Exp. Ophthalmol. 233:617-620.

33. Chihara, E., T. Matsuoka, Y. Ogura, and M. Matsumura. 1993. Retinal nerve fiber layer defect as an early manifestation of diabetic retinopathy. Ophthalmology. 100:1147-1151.

34. Enea, N.A., T.M. Hollis, J.A. Kern, and T.W. Gardner. 1989. Histamine $\mathrm{H}_{1}$ receptors mediate increased blood-retinal barrier permeability in experimental diabetes. Arch. Ophthalmol. 107:270-274.

35. Hammes, H.-P., H.J. Federoff, and M. Brownlee. 1995. Nerve growth factor prevents both neuroretinal programmed cell death and capillary pathology in experimental diabetes. Mol. Med. 1:1076-1551.

36. Alexiades, M.R., and C. Cepko. 1996. Quantitative analysis of proliferation and cell cycle length during development of the rat retina. Dev. Dyn. 250: 293-307.

37. Raff, M.C., B.A. Barres, J.F. Burne, H.S. Coles, Y. Ishizaki, and M.D. Jacobson. 1993. Programmed cell death and the control of cell survival: lessons from the nervous system. Science. 262:695-700.

38. Voll, C.L., and R.N. Auer. 1991. Insulin attenuates ischemic brain dam- 
age independent of its hypoglycemic effect. J. Cerebral Blood Flow Metab. 11: $1006-1014$.

39. Jonas, E.A., R.J. Knox, T.C.M. Smith, N.L. Wayne, J.A. Connor, and L.K. Kaczmarek. 1997. Regulation by insulin of a unique neuronal $\mathrm{Ca}^{2+}$ pool and of neuropeptide secretion. Nature. 385:343-346.

40. Yamaguchi, T., K. Keino, and J. Fukuda. 1995. The effect of insulin and insulin-like growth factor-1 on the expression of calretinin and calbidin D-28k in rat embryonic neurons in culture. Neurochem. Int. 26:255-262.

41. Barres, B.A., R. Schnid, M. Sendnter, and M.C. Raff. 1993. Multiple extracellular signals are required for long-term oligodendrocyte survival. Development. 118:283-295.

42. Meyer-Franke, A., M.R. Kaplan, F.W. Pfrieger, and B.A. Barres. 1995.
Characterization of the signaling interactions that promote the survival and growth of developing retinal ganglion cells in culture. Neuron. 15:805-819.

43. Barber, A.J., S. Dabulis, S. Khin, E. Lieth, and T.W. Gardner. 1997. Diabetic conditions cause apoptosis in retinal endothelial cells. Invest. Ophthalmol. Vis. Sci. (suppl) 37:S771. (Abstr.)

44. Janzer, R.C., and M.C. Raff. 1987. Astrocytes induce blood-brain barrier properties in endothelial cells. Nature. 325:253-257.

45. Gardner, T.W., E. Lieth, S.A. Khin, A.J. Barber, T. Lesher, D.J. Bonsall, K. Rice, and W.A. Brennan. 1997. Astrocytes increase barrier properties and ZO-1 expression in retinal vascular endothelial cells. Invest. Ophthalmol. Vis. Sci. 38:2423-2427. 